Aus der II. chirurgischen Universitätsklinik in Wien (Vorstand: Hofrat Prof. J. H o ch e n e g g).

\title{
Zur Kenntnis der Hernia obturatoria und der Hernia cruralis "praevascularis“.
}

Von Dr. Alfred Zinner, gew. Operationszögling der Klinik.

(Mit 4 Abbildungen.)

Der ersten Beschreibung der Hernia obturatoria durch Le N a i re (II) im Jahre I $7 \mathbf{I} 8$ sind im Laufe der Jahre zahlreiche kasuistische Mitteilungen von Brüchen des eirunden Loches gefolgt, so daß Graser (6) im Handbuch der Klinischen Chirurgie von 200 Fällen dieser seltenen Bruchart berichten konnte, denen $\mathrm{Tsch} \mathrm{marke} \mathrm{(I \gamma )}$ in jüngster Zeit drei weitere hinzufügte.

Wenn ich im folgenden einen Fall von eingeklemmter Hernia obturatoria eingehender beschreibe, so sehe ich mich dazu einmal durch den Umstand veranlaßt, daß dieser Fall, was Geschlecht und Alter, Symptomatologie und Diagnosestellung und den unglücklichen Ausgang anlangt, ein typischer Schulfall ist, ferner dadurch, daß trotz ausführlicher monographischer Bearbeitung dieses Themas durch R. Fischer (4) Englisch (I) u. a. eine übersichtliche graphische Darstellung der anatomischen Verhältnisse der Hernia obturatoria fehlt, endlich dadurch, daß in unserem Falle diese Hernie mit einer selten vorkommenden Form von Schenkelbruch vergesellschaftet war.

\section{Krankengeschichte.}

Die 47 jährige Schuhmachersgattin M. K. war angeblich nie ernstlich krank. Sie hat zehnmal geboren, letzter Partus vor $61 \% 2$ Jahren. Seit I Jahre Menopause. Seit dieser Zeit traten bei Frau K. in langen Intervallen sehr heftige, krampfartige Schmerzen in dem rechten Oberschenkel auf. Dieselben waren immer an der gleichen Stelle lokalisiert und waren so heftig, daß die Pat. nicht gehen konnte. Die 
Anfälle gingen jedesmal nach einigen Stunden ohne jede Medikation vorüber. Am 23. VI. 1908 hatte die Pat. wieder einen typischen Anfall, nur wollte derselbe diesmal nicht aufhören. Der herbeigerufene Arzt verordnete Pulver, worauf die Schmerzen nachließen. Am nächsten Tage klagte die Frau über Mattigkeit und Fieber. Singultus und Erbrechen stellten sich ein. Die beiden behandelnden Ärzte konnten keine Diagnose stellen. Am dritten Tag wurde die Frau ins Allgemeine Krankenhaus gebracht, wo $1 \mathrm{e} 1 / 2$ Stunde nach der Einlieferung starb.

Bei der von Prof. $\mathrm{K}$ o l i s k o am 26 . VI. vorgenommenen (sanitätspolizeilichen) Obduktion wurde als Todesursache Darmlähmung infolge von Einklemmung eines Darmwandbruches im rechtsseitigen verstopften Loche (Hernia obturatoria dextra incarcerata Littre) aufgedeckt. Außer dieser Hernie fand sich beiderseits ein Schenkelbruch und eine $6 \mathrm{~cm}$ lange rechtsseitige Labialhernie (s. Fig. I).

Typisch ist in unserem Fall zunächst die Tatsache, daß er eine Frau betrifft. Erfahrungsgemäß neigen dieselben vielmehr dazu als die Männer, da ihr Becken niedriger und breiter ist, und das Foramen obturatum einen breiteren Horizontaldurchmesser hat als beim Mann $(4: 3,25 \mathrm{~cm})$, daher ist auch der Sulcus, bzw. Canalis obturatorius breiter. Demzufolge überwiegt das weibliche Geschlecht ganz außerordentlich. Nach Englis ch (l. c.) kamen auf 96 Weiber I 8 Männer, was einem Verhältnis von $8:$ I entspricht. Die größte Zahl der Fälle steht nach $F$ is cher (l.c.) zwischen dem 40. und 6o. Lebensjahr (unsere Patientin war 47 Jahre alt).

Als eine der Hauptursachen der Entstehung der Hernia obturatoria wurden von Vinson, Garangeod $(I 8,5)$ wiederholte Schwangerschaften bezeichnet, da infolge der Zerrung eine Erschlaffung des Beckenbindegewebes zurückbleibt. Unsere Pat. hat romal geboren.

In sehr präziser Weise trat das Howship-Rombergsche Phänomen ( 7, I6) neuralgische Schmerzen und Krämpfe im Bereiche des Nervus obturatorius zntage. Kenntnis dieses Phänomens hätte zu der richtigen Diagnose führen müssen. So aber mußte die Patientin das traurige Schicksal der meisten ihrer Leidensgenossinnen teilen. ( $\mathrm{E} n \mathrm{~g} \mathrm{l}$ is $\mathrm{ch}$ gibt an, daß in Io7 Fällen bloß $25 \mathrm{mal}$ die richtige Diagnose gestellt wurde; 60 mal wurde die Art des Bruches erst bei der Autopsie erkannt.)

Bevor wir auf die nähere Beschreibung unseres Falles eingehen, wollen wir kurz die anatomischen Verhältnisse rekapitulieren. 
Die obere, innere und äußere Wand des Canalis obturatorius wird durch Knochen gebildet (oben durch den Sulcus obturatorius) die untere durch eine Bindegewebsmembran, die als eine Ausstülpung der Fascia pelvis aufzufassen ist, oben und seitwärts mit dem Periost verwachsen ist, vorne und rückwärts durch den oberen freien bandartigen Rand der Membrana obturatoria externa und interna begrenzt wird. Die das Foramen obturatum größenteils verschließende Membrana obturatoria teilt sich nämlich in ihrem oberen Anteil in zwei Lamellen, von denen die eine als Membrana obturatoria externa sich an der äußeren, die innere als Membrana obturatoria interna sich an der inneren Fläche des Schambeins ansetzt. Der Raum zwischen beiden ist von Fettgewebe, Gefäß- und Nervenzweigen ausgefüllt. Der Canalis obturatorius wird von dem Nervus obturatorius, der sich in einen Ramus superficialis und profundus spaltet und der Arteria und Vena obturatoria durchzogen.

Den anatomischen Verhältnissen entsprechend kann man daher mit v. M e ye r (I2) folgende Arten der Hernia obturatoria unterscheiden.

A. eine Hernia incompleta s. intracanalis, wenn der Bruchsack im Kanal bleibt,

B. eine Hernia completa, wenn der Bruchsack die äußere Offnung des Kanals passiert, unterscheiden.

Da der Nervus obturatorius allseitig von Fett umschlossen ist, erfolgt die Entwicklung der Hernie vorzüglich längs der Äste desselben. Nach H. v. M e y e r lassen sich zweckmäßig folgende drei Formen auseinanderhalten:

I. Eine Hernia obturatoria anterior, die dem Ramus anterior s. superficialis des Nerven folgt. Da dieser Ast der größte ist und am reichlichsten von Fett umgeben ist, ist diese Form der Hernia obturatoria die häufigste. Die Bruchgeschwulst liegt dann unter dem Musculus pectineus.

2. Die Hernie begleitet den Ramus posterior oder profundus des Nervus obturatorius, Hernia obturatoria posterior. Ist der Bruchsack klein, wie in unserem Fall, so reicht er nur bis zu dem oberen Rand des Musculus obturator externus, ist er größer, so tritt er zwischen der oberen und mittleren 
Portion dieses Muskels hervor und kommt dann ebenfalls unter den Musculus pectineus zu liegen.

3. Die Hernie verbleibt zwischen den beiden Membranae obturantes. Diese Form ist die seltenste.

In unserem Falle ergab sich folgendes:

A. Präparation des Bruchsackes von vorne (a u B e n) [s. Fig. 2].

Nach Durchtrennung der beiden Blätter der Fascia lata und des Musculus pectineus stößt man auf eine derbe Fascia, deren

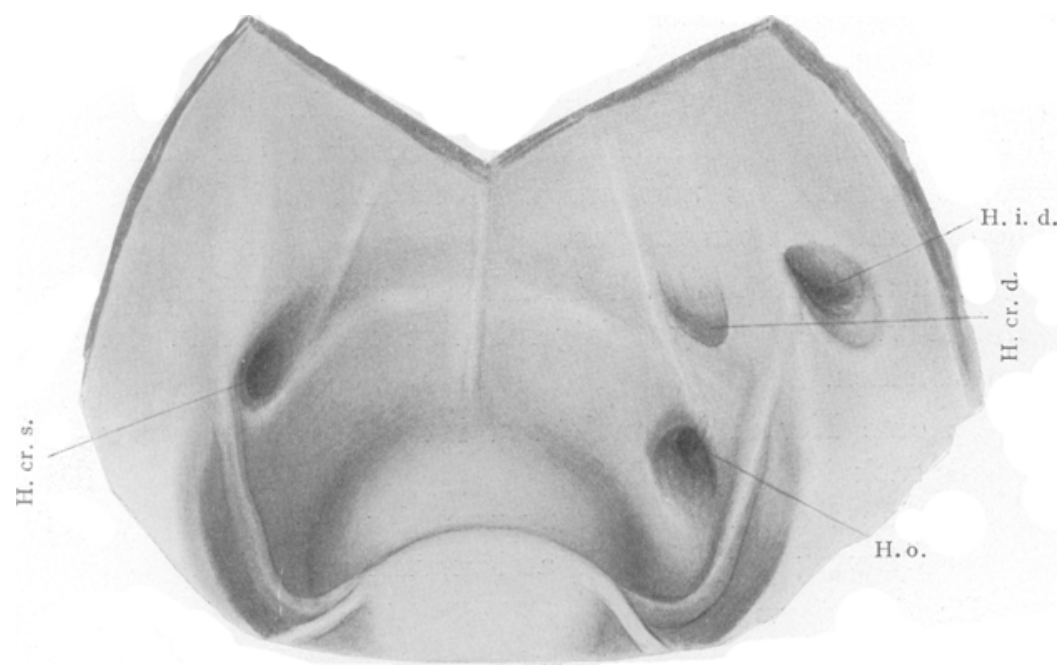

Fig. I.

Das Becken von innen. - H. cr. d. = Hernia cruralis dextra. - H. cr. s. = Hernia cruralis sinistra. - H. i. d. = Hernia inguinalis dextra. - H. o. = Hernia obturatoria.

craniales Ende sich an einem Sehnenstreifen des Os pubis ansetzt, und die, den Raum zwischen horizontalem Schambeinast und dem freien Rand des Musculus adductor brevis überbrückend, kaudal auf diesen Muskel übergeht. Dieses Facienblatt, Fascia musculi adductoris brevis, deckt alle in der Tiefe liegenden Gebilde $z u$, den Ramus superficialis des Nervus obturatorius sieht man durchschimmern. Nach Durchtrennung dieser Fascie wird der freie obere Rand des Musculus obturator externus, über den 
der oberflächliche Ast des Nervus obturatorius hinwegzieht, und die Apertura externa des Canalis obturatorius sichtbar. Aus dieser sieht man einen kirschgroßen Tumor hervorragen, über dessen vordere Fläche, innig mit ihm verwachsen, der tiefe Ast des Nervus obturatorius zieht, um sich an der Hinterseite des Musculus obturator externus in diesen einzusenken.

Es handelt sich daher in unserem Fall um eine Hernia obturatoria posterior.

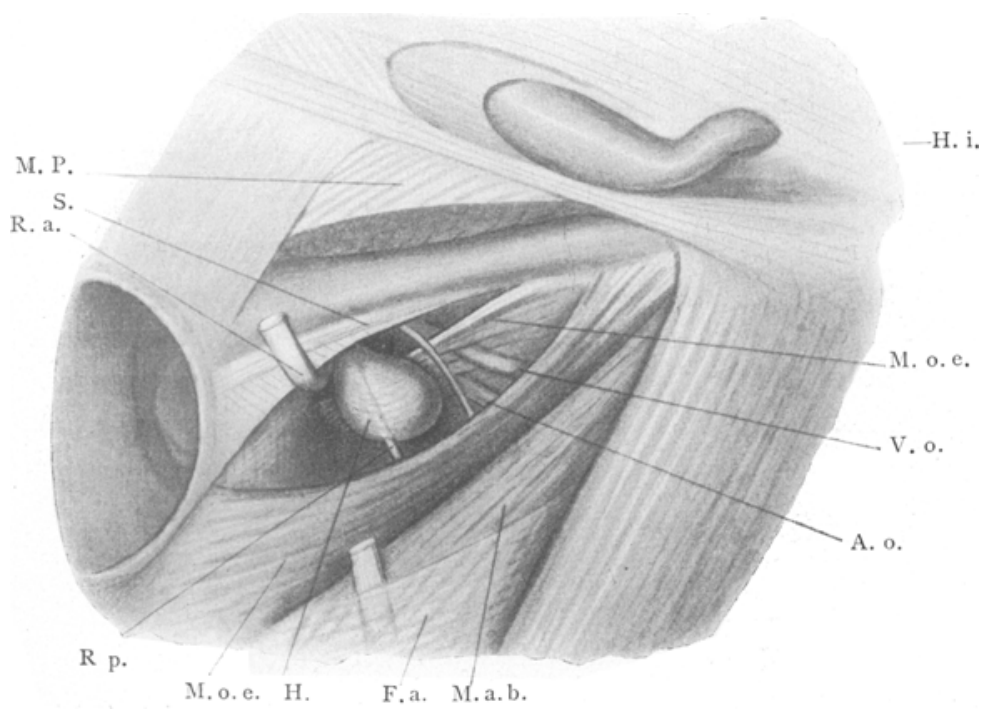

liig. 2.

M. $\mathrm{P}$. = Musc. pectineus. - M. a. b. = Musc. adductor brevis. - M. o. c. $=$ Mluse. obturator externus. - F. a. $=$ Fascia Musc. adductoris brevis. - M. $=$ Hembrana obturatoria externa. $-\mathrm{S}$. $=$ Sehnenstreifen am Os pubis. $-\mathrm{A} . \mathrm{o}=$ Arteria obturatoria. - V. o. = Vena obturatoria. - R. a. = Ramus anterior (Nervi obturatorii). - R. $\mathrm{p}=$ Ramus posterior (Nervi obturatorii). $-\mathrm{H} .=$ Bruchsack. - H. i. = Hernia inguinalis.

Zieht man den oberen Rand des Musculus obturator externus nach abwärts und löst man die obersten Fasern dieses Muskels von der Membrana obturatoria externa $a b$, so sieht man den freien oberen Rand dieser Membran, die von der Arteria und Vena obturatoria durchbohrt wird, und zwar liegt die Arterie lateral, die Vene medial. 
B. Präparation von innen (s. Fig. 3).

Das Bauchfell wurde in einiger Entfernung von der Bruchpforte zirkulär umschnitten und zurückpräpariert; auf diese Weise kommt die Fascia pelvis zur Ansicht. Diese wurde ebenfalls zirkulär umschnitten und gegen die Mündung des Canalis obturatorius zurückpräpariert, was mühelos gelingt. Die Fascia pelvis bildet um den Bruchsackhals, ihn außen umgebend, einen derben wohl ausgebildeten Bindegewebstrichter, der in den Canalis ob-

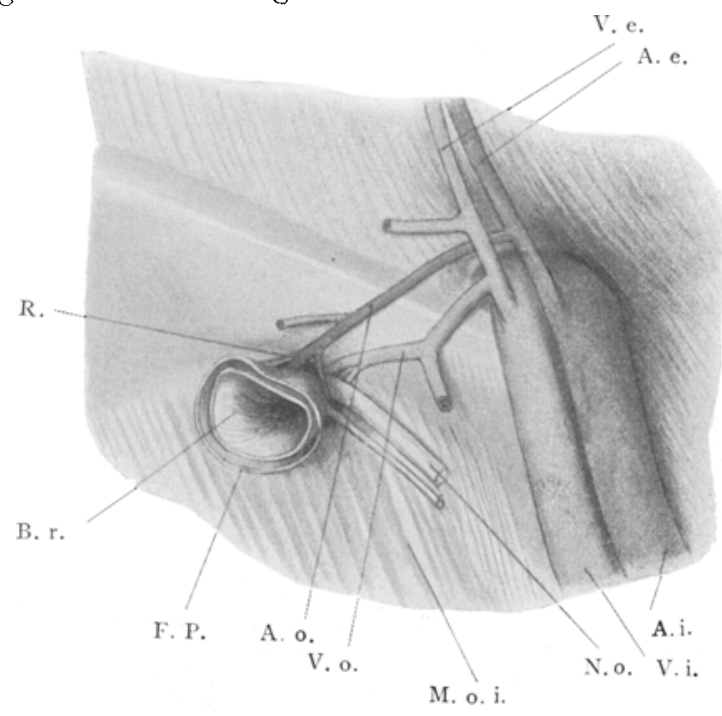

Fig. 3.

Br. = Bruchsackhals. - F. P. = Der von der Fascia pelvis gebildete Trichter. A. i. = Arteria iliaca externa. - V. i. = Vena iliaco externa. - A. e. = Arteria epigastrica inf. - V. e. = Vena epigastrica inf. - A. $0 .=$ Arteria obturatoria. V. o. = Vena obturatoria. - $\mathrm{R}$. = Ramus muscularis d. Art. obturatoria. M. o. i. $=$ M. obturator internus. - N. o. $=$ Nervus obturatorius.

turatorius eindringt und innerhalb desselben mit dem Periost und den Vasa obturatoria sehr innig verbunden ist. Durch die Ablösung der Fascia pelvis werden die Vasa obturatoria sichtbar, die beiderseits aus der Arteria bzw. Vena epigastrica hervorgehen.

An der Apertura interna des Canalis obturatorius besteht folgendes topographische Verhältnis. Am meisten lateral liegt die Vene, dann der Nerv, am meisten medial die Arteria obturatoria. 
An der Apertura externa liegt der Nerv lateral und vorne, dann folgt die Arterie, unten innen, am meisten medial, die Vene. Es hat also im Kanal eine völlige Umlagerung der Gefäß- und Nervenäste stattgefunden (s. die Fälle von $\mathrm{Scholz}, \mathrm{De}$ m e a u x usw. usw. (2, I7)).

Auffallend häufig erscheint der Hüftlochbruch mit anderen Brüchen, insbesondere Schenkelbrüchen kombiniert. (Daher ist es vorgekommen, daß Operateure wie $\mathrm{z}$. B. N u B b a u m (r4) eine

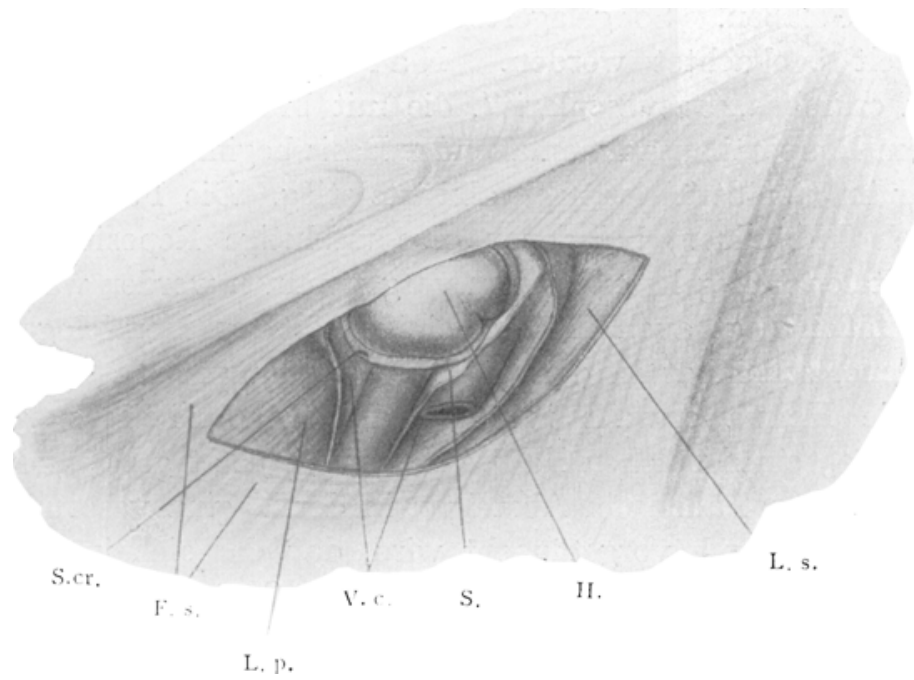

Fig. 4.

H. = Bruchsack. - F. s. = Fascia superficialis. - L. s. = Lamina superficialis fasciae latac. - L. p. = Lamina profunda fasciae latae. - S. = Septum vaginae vasorum. - S. cr. = Septum crurale Cloqueti z. T. reseziert und mit einem Haken abgezogen. - - V. c. = Vagina vasorum communis (geöffnet).

Schenkelhernie operierten und die Autopsie dann mit der brandigen Schlinge im Canalis obturatorius überraschte. Ähnlich erging es $\mathrm{Nica}$ ise s. Sch midt (I5).)

Auch in unserem Fall waren neben einer $6 \mathrm{~cm}$ langen rechtsseitigen Labialhernie beiderseits Cruralhernien vorhanden, von denen die rechte klein ist und bloß die Fingerkuppe faßt, während die linke größere eine ziemlich selten vorkommende Form des Schenkelbruchs repräsentiert (s. Fig. 4). Der Bruchsack verläßt an 
normaler Stelle, medial von der Arteria epigastrica die Bauchhöhle Während nun ein gewöhnlicher Schenkelbruch medial von der Vena femoralis zu liegen kommt - die Schenkel venewird daher niemals durch die Schenkelhernie komprimiert, „weshalb man bei Schenkelbrüchen weder ein dauerndes noch vorübergehendes Ödem der unteren Extremität beobachtet" (M a idl Io) - befindet sich in unserem Fall der Bruchsack, dessen größte Breite von $3 \frac{1 / 2}{\mathrm{~cm}}$ mit dem vorderen Band des Ligamentum Pouparti zusammenfällt, im Gefäßtrichter, der Vagina vasorum communis, vor den Gefäßen. So gelagerte Schenkelhernien sind nur ganz vereinzelt beobachtet worden. $\mathrm{Narath}$ (I3) beschrieb eine Hernia cruralis ,praevascularis“, die mit breiter, mit dem Ligamentum Pouparti zusammenfallender Basis beginnend, einige Zentimeter im Gefäßtrichter nach abwärts stieg. Die Fälle betrafen sämtlich Individuen, bei denen eine unblutige Reposition einer kongenitalen Hüftgelenksluxation vorgenommen worden war. Vor den Gefäßen liegt auch die von einigen älteren Herniologen beschriebene, scheinbar in der Vagina vasorum propria selbst absteigende Hernia cruralis intravaginalis $[\mathrm{Fabricius}$ (3) erwähnt I895 einen solchen Fall]. H y r 1 (8) hat gezeigt, daß in diesen seltenen Fällen ein Absteigen in der Vagina vasorum propria selbst dadurch vorgetäuscht wird, daß das sich in den Gefäßtrichter ,,einschiebende Eingeweide das Septum crurale nicht vordrängt, sondern dieses von der Schenkelgefäßscheide eine Strecke weit gleichsam lospräpariert". In unserem Fall nun umgab das vorgebuchtete Septum curale als leicht darzustellende Membran den ganzen Bruchsack, um sich in normaler Weise unter dem Ligamentum Pouparti mit der Vagina vasorum propria zu vereinigen. Die Erklärung für die abnorme Lagerung unserer Hernie ist daher in einem anderen Umstand zu suchen. L in h a r t (9) hat gezeigt, daß die oft sehr derbe innere Wand des Gefäßtrichters sich in einem variablen Winkel mit dem Ligamentum Pouparti resp. Gimbernati vereinigen kann. Ist dieser Winkel spitz, so muß, die Unnachgiebigkeit der Innenwand der Vagina vasorum communis vorausgesetzt, eine durch den Annulus femoralis hervordringende Hernie etwas nach außen abgelenkt werden und daher vor den GefäBen zu liegen kommen. Auf dieseWeise dürfte sich in unserem und ähnlichen Fällen die Hernia cruralis ,praevascularis" erklären lassen. 
Erwägen wir, um noch einmal auf die Hernia obturatoria zurückzugreifen, daß nur sehr selten eine palpable große Bruchgeschwulst sich vorfindet, da $B$ in den meisten Fällen von Incarceration eines Hüftlochbruches Darmwandbrüche vorliegen und man sich daher, wegen der Enge der Bruchpforte, beim Bloßlegen des Bruchsackes von außen nur sehr schwer über die fixierte Darmschlinge wird orientieren können, so hat die Operation der Wahl bei bestehendem Verdacht einer Hernia obturatoria incarcerata die Laparotomie zu sein ${ }^{\mathbf{1}}$. Desgleichen wird man sich, die traurigen Erfahrungen von $\mathrm{N}$ i c a is e, $\mathrm{N} \mathrm{u} \mathrm{B} \mathrm{b} \mathrm{a} \mathrm{u} \mathrm{m} \mathrm{(l.} \mathrm{c.)} \mathrm{usw.}$ benützend, - zumal bei Frauen - zur Laparotomie entschließen, wenn bei deutlich vorhandenen Einklemmungssymptomen der anatomische Befund eines bloBgelegten Leisten- oder Schenkelbruchs ein unbefriedigender ist.

Herrn Hofrat $\mathrm{K}$ o lis $\mathrm{k}$ o danke ich herzlichst für die Überlassung des Falles.

\section{Literaturverzeichnis.}

I. E $\mathrm{ng} 1$ is ch, A., Über Hernia obturatoria. Wien I892.

2. De m a ux, Bulletins de la soc. anatomique, I839, zit. nach Englisch (1. c.).

3. Fabricius, J., Über die operative Behandlung von Cruralhernien. Wien. klin. Wochenschr. 1895 , Nr. 31 u. 32.

4. F i s c h e r, R., Beiträge zur Lehre über die Hernia obturatoria. Leipzig 1856.

5. Croissant de Garangeot, Mémoires sur plusieurs hernies singulières. Mémoire de l'académie royale de Chirurgic I787, zit. nach Englis ch.

6. Graser, Handbuch der klinischen Chirurgie ( $v$. Bergmann, Br uns, Mikulicz), 3. Bd.

7. How ship, Practical Remarks on the discrimination and Appearances of the surgical diseases, I840, zit. nach $\mathrm{Eng}$ l is ch (1. c.).

8. Hy rtl, J., Lehrbuch der topographischen Anatomie I87I, Bd. II, S. 557.

9. Linhart, W., Vorlesungen über Unterleibshernien. Würzburg $\mathbf{1} 866$.

ro. Maidl, K., Die Lehre von den Unterleibsbrüchen, Wien I898, S. 64. (Der spez. Chirurgie, I, Bd.)

II. Le $\mathrm{Maire}$, I7I8, zit. nach $\mathrm{Englisch}$ (1. c.).

I) Nachtrag bei der Korrektur: Zu dem gleichen Schluß kornmt in einer während der Drucklegung dieser Arbeit erschienenen Mitteilung Gelpke (diese Zeitschrift, Bd. 21, H. I-3, S. 239).

Deutsche Zeitschrift f. Chirurgie. IO3. Bd. 
12. v. M e y e $\mathrm{r}$, H., Lehrbuch der Anatomie des Menschen, 3. Auflage. Leipzig 1873 , S. 752 u. 753 .

3. Narath, Eigenartige Hernia curalis nach Einrichtung von Luxatio fem. cong. Arch. f. klin. Chir. r899, Bd. 59.

14. $\mathrm{N}$ i c a is e (zit. nach J. R o se). Weitere Betrachtungen über den Bruchschnitt, Deutsche Zeitschr. f. Chir., Bd. 35.

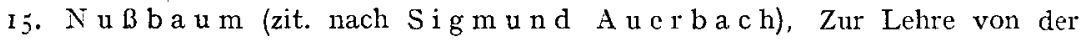
Hernia obturatoria. Diss, München 1890 .

r6. $\mathrm{Romberg}$, in Dieffenbachs operativer Chirurgie, Bd. 2, S. 625.

17. Scholz, Ein Fall von Hernia foraminis ovalis. Wochenbl. d. k. k. Gesellschaft d. Arzte in Wien I860.

18. T s c h marke. P. Deutsche Zeitschr. f. Chir., Bd. 45, S. 49.

I9. Vi n s o n, De la Hernie souspruibienne, Thèse de Paris 1844. 